\title{
Model of pulse separation in the gamma light curve of the Vela pulsar
}

\author{
Jarosław Dyks and Bronisław Rudak \\ N. Copernicus Astronomical Center, Rabiańska 8, 87100 Toruń, Poland
}

\begin{abstract}
Within the framework of a single polar cap model we calculate the energy dependence of phase separation between two peaks in the gamma-ray lightcurve of the Vela pulsar. Results are confronted with EGRET data (Kanbach 1999).
\end{abstract}

\section{The Model and Results}

We have used the polar cap model with most ingredients as postulated by Daugherty \& Harding (1982). High-energy emission in the model is a superposition of curvature radiation (CR) of primary electrons (beam particles) and synchrotron radiation (SR) of secondary particles ( $e^{ \pm}$pairs) created via magnetic absorption of photons. We have considered four cases (A, B, C, and D) with the spin period of the Vela pulsar and the surface magnetic field strength $B_{\mathrm{pc}} \sim 10^{12} \mathrm{G}$.

In models $\mathrm{A}$ and $\mathrm{B}$, primary electrons are injected at initial height $h_{\text {init }}$ equal to 0 and $1 R_{\mathrm{ns}}$ above the star surface with ultrarelativistic energy $E_{\text {init }}$ equal to 8.7 and $20 \mathrm{TeV}$, respectively. In model $\mathrm{C}, h_{\text {init }}=2 R_{\mathrm{ns}}$, and the electrons are accelerated from rest by a constant electric field $\mathcal{E}_{\|}=2.55 \times 10^{7} \mathrm{Vcm}^{-1}$ present over the scale height $0.6 R_{\mathrm{ns}}$. A hollow cone geometry is assumed in models $\mathrm{A}$, $\mathrm{B}$, and $\mathrm{C}$, i.e. the electrons move only along field lines from the outer rim of the polar cap. The angle between the spin axis $\vec{\Omega}$ and the magnetic moment $\vec{\mu}$ is set to $3.0,5.0$, and 10.0 degrees, respectively for $\mathrm{A}, \mathrm{B}$, and C. Model D differs from model A only in that a uniform electron distribution over the entire polar cap surface has been assumed for it (filled column geometry).

Results of the calculations are summarized in Figure 1. In the low-energy domain (i.e. below a few $\mathrm{GeV}$ ) the peak separation $\Delta^{\text {peak }}$ either slightly decreases or remains constant with increasing energy $\epsilon$ over a wide energy range, but in each case is consistent with Kanbach (1999). In this domain the synchrotron component either dominates over the curvature component or is equally important. Consequently, the behaviour of $\Delta^{\text {peak }}$ is affected by directional and spectral properties of the SR emission. The slight decrease of $\Delta^{\text {peak }}$ with increasing $\epsilon$ (model A) occurs only under special conditions discussed in detail in Dyks \& Rudak (2000, in preparation).

In the high-energy domain (i.e. above a few $\mathrm{GeV}$ ) the phase separation $\Delta^{\text {peak }}$ undergoes an abrupt turnover, and changes dramatically. It increases in our hollow-cone models (A, B, and $\mathrm{C}$ ) and decreases in the filled-column model (D) at a rate $\sim 0.28$ phase per decade of photon energy. This effect is due to magnetic absorption $\left(\gamma \vec{B} \rightarrow e^{ \pm}\right)$in the magnetosphere (see also Miyazaki 

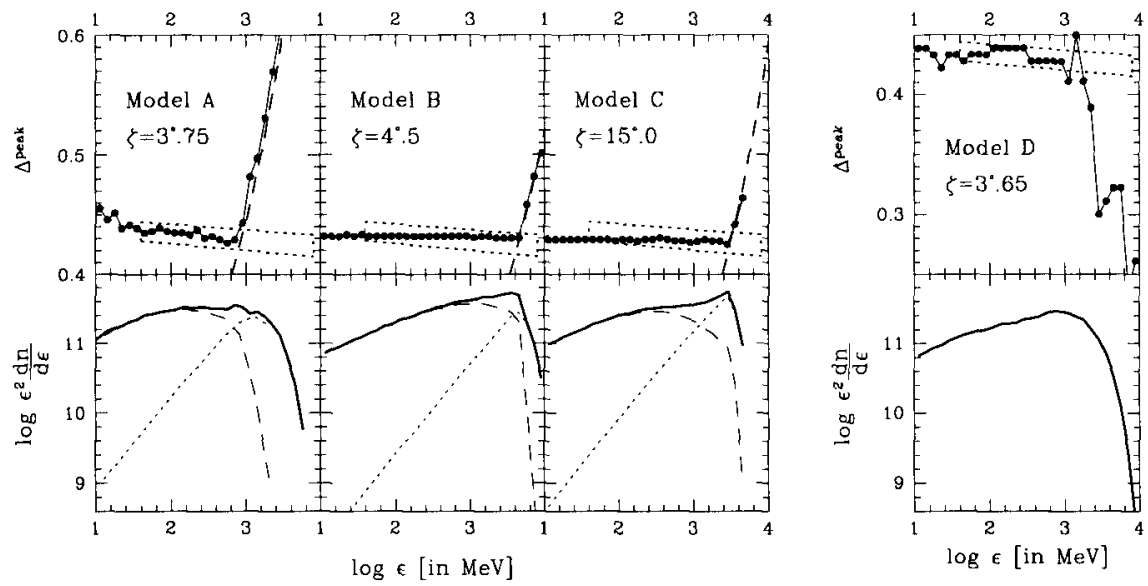

Figure 1. Upper panels: Phase separation $\Delta^{\text {peak }}$ versus photon energy $\epsilon$ of the emission peaks found with Monte Carlo calculations for models A, B, C and D (connected dots). The assumed values of the angle $\zeta$ between $\vec{\Omega}$ and the line of sight are indicated. The dotted parallelogram marks schematically the results of Kanbach (1999) for the Vela pulsar. The steep dashed lines in the three left-hand side panels are the approximate analytical solutions for $\Delta^{\text {peak }}$ determined by magnetic absorption effects $\left(\gamma \vec{B} \rightarrow e^{ \pm}\right)$.

Lower panels: Energy output per logarithmic energy bandwidth at the first peak as a function of photon energy $\epsilon$ for models $A, B, C$ and $\mathrm{D}$ (solid). The synchrotron (long dashed) and the curvature (short dashed) components are marked for models A, B, and C. Units on the vertical axis are arbitrary.

\& Takahara 1997), and may be reproduced with good accuracy with simple analytical approach. If observed with next generation gamma-ray missions, such turnover in $\Delta^{\text {peak }}$ at a few $\mathrm{GeV}$ would be an important signature of polar cap activity in gamma-ray pulsars. Moreover, it would support the notion that highenergy cutoffs in gamma-ray spectra of pulsars are due to magnetic absorption.

\section{References}

Daugherty, J. K., Harding A. K. 1982, ApJ, 252, 337

Kanbach G. 1999, in Proc. of 3rd INTEGRAL Workshop, in press Miyazaki J., Takahara F. 1997, MNRAS, 290, 49 